\title{
Diagnostic Value of Diffusion Weighted Magnetic Resonance Imaging in Evaluation of Metastatic Neck Lymph Nodes in Head and Neck Cancer: A Sample of Iranian Patient
}

\author{
Fatemeh Alamolhoda ${ }^{1}$, Fariborz Faeghi ${ }^{1 *}$, Mohsen Bakhshandeh ${ }^{1}$, Aslan \\ Ahmadi $^{2}$, Morteza Sanei Taheri ${ }^{3}$, Sahar Abbasi ${ }^{3}$
}

\begin{abstract}
Objective: To evaluate the diagnostic value of DWI in assessment of metastatic neck lymph node in a sample of Iranian patients with Head and Neck cancer. Methods: 25 patients with 80 neck lymph nodes were analyzed using $1.5 \mathrm{~T}$ MRI. DWI was performed with b values of 0 and $1,000 \mathrm{~s} / \mathrm{mm}^{2}$. Short axis diameter and ADC values (min, max and mean) were calculated for metastatic and non-metastatic neck LNs and results were compared with histopathological findings. The optimal ADC thresholds were analyzed using receiver coefficient characteristic (ROC) curves for discriminating between metastatic and benign neck lymph nodes. Result: Histopathological findings revealed that there are $45 \%(n=36)$ metastatic and 55\% $(n=44)$ non-metastatic neck LNs respectively. There was no statistically significant difference in short axis diameter between the two groups $(\mathrm{p}=0.346)$. However, The ADC values of metastatic neck LNs were significantly lower than those of non-metastatic neck LNs $(\mathrm{p}<0.001) ; 0.90 \pm 0.10 \times 10^{-3} \mathrm{~mm}^{2} / \mathrm{s}$ vs $1.06 \pm 0.12 \times 10^{-3}$ $\mathrm{mm}^{2} / \mathrm{s}$ ( ADC mean ), $0.78 \pm 0.08 \times 10^{-3} \mathrm{~mm}^{2} / \mathrm{s}$ vs $0.92 \pm 0.20 \times 10^{-3} \mathrm{~mm}^{2} / \mathrm{s}($ ADC min $)$ and $1.02 \pm 0.12 \times 10^{-3} \mathrm{~mm}^{2} / \mathrm{s}$ vs $1.24 \pm 0.15 \times 10^{-3} \mathrm{~mm}^{2} / \mathrm{s}$ (ADC max ). The optimal mean ADC threshold value was equal to $0.996 \times 10^{-3} \mathrm{~mm}^{2} / \mathrm{s}$ for differentiating malignant from benign lymph nodes with sensitivity, specificity and accuracy of $80.56 \%, 77.27 \%$ and $71.59 \%$ respectively. Conclusion: MR diffusion imaging and ADC values as a non-invasive technique can assess metastatic neck LNs in head and neck cancer with higher sensitivity, specificity and accuracy.
\end{abstract}

Keywords: DWI-MRI- neck lymph nodes- Head and neck cancer- metastasis

Asian Pac J Cancer Prev, 20 (6), 1789-1795

\section{Introduction}

Head and neck cancer is placed on the fifth most common cancer worldwide, accounting for about $3 \%$ to $4 \%$ of all malignancies. Lymph node metastases are found in more than half of these patients and demonstrate a most important factor in predicting outcome and selecting treatment (Vandecaveye et al., 2012; Noij et al., 2015; Dudau et al., 2014; Kim et al., 2009; Mack et al., 2008). It has been reported that the presence of a single metastatic node decreases the rate of survival by $50 \%$ and the rate of affecting to bilateral disease by an additional 50\% (Dudau et al., 2014). Differentiation of benign and malignant neck lymph nodes is important especially in the presence of head and neck malignancy for staging, treatment planning and follow-up of cancer (Vandecaveye et al., 2009; de Bondt et al., 2009; Ali, 2012; Dawood et al., 2014; ElSaid et al., 2014; Ali and El Hariri, 2017). Small lymph nodes with a maximum short axial diameter below $10 \mathrm{~mm}$ represent one of the most challenging problems for radiologists (de Bondt et al., 2009; ElSaid et al., 2014). Conventional imaging including: Ultrasound (US), computed tomography (CT) and magnetic resonance imaging (MRI) relies on the morphological criteria of the lymph node including maximum short axis diameter, lymph node hilum loss and necrosis along with heterogeneous pattern of post contrast enhancement and perinodal infiltration. These image methods can diagnose cervical lymphadenopathy, but, they cannot differentiate benign lymph nodes from malignant ones accurately (Ali, 2012; Dawood et al., 2014; and El Hariri, 2017; Razek et al., 2006; Holzapfel et al., 2009; Salem et al., 2014; Liang et al., 2017). Another imaging modalities such as single photon emission computed tomography (SPECT) and positron emission tomography (PET) can be helpful in this differentiation but they are expensive and are limited to low spatial resolution, as well as false diagnosis of physiological and inflammatory 
lymph nodes( Ali, 2012; Ali and El Hariri, 2017; Razek et al., 2006; Holzapfel et al., 2009). Ultrasound guided fine needle aspiration cytology (USgFNAC) can help in characterizing cervical lymph nodes, but this method is invasive with false negative results as this is dependent on the operator ( Ali, 2012; Ali and El Hariri, 2017; Razek et al., 2006; Holzapfel et al., 2009; Liang et al., 2017).

Diffusion-weighted MRI (DWI) is a non-invasive technique that provides quantitative information of the molecular diffusion in several compartments. Any architectural changes in the proportion of extracellular to intracellular water protons like metastases in lymph nodes will alter the apparent diffusion coefficient of the tissue. So, DWI provides information for identification and characterization of different tissues and lesions (de Bondt et al., 2009; Dawood et al., 2014; ElSaid et al., 2014; Perrone et al., 2011; Zhang et al., 2013; Ragheb et al., 2014). The aim of this study is to evaluate the diagnostic value of DWI in assessment of metastatic neck lymph node in a sample of Iranian patients with Head and Neck cancer.

\section{Materials and Methods}

\section{Patients}

This study was conducted prospectively from 3 May 2017 to 10 January 2018 at the MRI department of Shohadaye Tajrish Hospital, and was included 25 consecutive patients with histologically proven Head and neck cancer and with enlarged neck nodes. According to the histopathological diagnoses, the patients were included as 9 patients with larynx cancer, 6 patients with Thyroid cancer, 4 patients with pharynx cancer, 3 patients with tongue cancer and 3 patients with parotid cancer. None of these patients had received neck clearance surgery, biopsy, chemotherapy or radiotherapy for neck LNs before MRI examination. Patients' age ranged between 17-77 years old and the affected side was located in the left side in 3 patients, in the right side in 3 patients and there was bilateral affection in 19 cases. After an MRI examination (lasting for 2 weeks from 2 to 15 days) all patients underwent neck lymph node surgical and received a definite pathological diagnosis. In total, 80 lymph nodes, 44 benign nodes, and 36 metastatic nodes were analyzed in this study. The study was approved by the local ethical committee of the university, and informed consent was obtained.

\section{MRI Examination}

Neck MRI examination were performed using a 1.5-T system (Magneto Avanto Siemens, Erlangen Germany). All patients were scanned with dedicated head and neck coils in the standard supine position.

\section{The MR protocol is as follows}

- T2W-FSE-sagittal: TR: $3200 \mathrm{~ms}$, TE: $80 \mathrm{~ms}$, slice

thickness: $4.5 \mathrm{~mm}$, Matrix: $320 \times 256$, NEX: 2

- STIR-coronal: TR: 4500 ms, TE: 40 ms, TI: $160 \mathrm{ms,}$ slice thickness: $4.5 \mathrm{~mm}$, matrix: $256 \times 256$, NEX: 2

- T2W-FSE-axial: TR: $4350 \mathrm{~ms}$, TE: $83 \mathrm{~ms}$, slice thickness: $5 \mathrm{~mm}$, Matrix: $320 \times 256$, NEX: 3

- T1W-FSE-axial: TR: $520 \mathrm{~ms}$, TE: $18 \mathrm{~ms}$, slice thickness: $5 \mathrm{~mm}$, Matrix: $320 \times 256$, NEX: 3

- T2W-axial-fat sat: TR: $3500 \mathrm{~ms}$, TE: $80 \mathrm{~ms}$, slice thickness: $5 \mathrm{~mm}$, Matrix: $320 \times 256$, NEX:3

Acquisition of diffusion-weighted images using single shot echo planar imaging (EPI) sequence was achieved in the axial plane. Diffusion gradient encoding was done in the three orthogonal planes $(\mathrm{X}, \mathrm{Y}, \mathrm{Z})$ with two $\mathrm{b}$ values $\mathrm{b}=0$ and $\mathrm{b}=1,000 \mathrm{~s} / \mathrm{mm}^{2}$. Imaging parameters for DWI were as follows: $\mathrm{TR}=4400 \mathrm{~ms}, \mathrm{TE}=88 \mathrm{~ms}, \mathrm{FA}=90$, Matrix size $=192 \times 192$, slice thickness $=4 \mathrm{~mm}, \mathrm{NEX}=6$, bandwidth $=1446 \mathrm{~Hz} /$ pixel. The ADC map was calculated automatically using a standard software imager in the MR system. The total scan time of DWI lasts 2 minutes. In addition, contrast-enhanced MRI scan is provided in sagittal, coronal and axial planes for each patient in order to confirm the localization of malignant tissue as well as any cysts or necrosis.

\section{Image analysis}

All MR images were read independently by two radiologists experienced in the head and neck MRI interpretation who did not any previous knowledge regarding the histopathological type of the nodes.

Conventional MR images were assessed for the evaluating morphological features of each lymph node. We selected the LNs with short axis larger than $5 \mathrm{~mm}$.

The final number of neck lymph nodes that were selected in the analysis was equal to 80 lymph nodes. In the case of patient with multiple lymph nodes (LNs), everyone was evaluated separately. The assessment of nodal ADC value includes drawing a region of interest (ROI) with greatest diameter for covering the pathologic node in all sections in which it was present and averaging the results. In this study, we excluded the necrotic areas from analysis to avoid presenting a false high ADC. All the ADC values were averaged from three-time measurement and were expressed as the mean \pm standard deviation. Three ADC values (min $\mathrm{ADC}$, max $\mathrm{ADC}$, mean $\mathrm{ADC}$ ) were calculated for all selected LNs.

Finally, MRI results were compared with the results of the pathological examination of the neck dissection samples.

\section{Statistical Analysis}

Statistical analysis of data was performed considering special characteristic (including apparent diffusion coefficient (ADC) value of the malignant tumors and benign lesions in head and neck cancer) in Statistical Package for Social Science (SPSS) version 25.

Descriptive Statistics was performed to display prevalence of the malignant and benign lesion in males and females and also to demonstrate mean age of them. The homogeneity of gender and age was tested between groups through chi-square independence test and independent T-test, respectively. Because of non-normal data distribution, the Mann-Whitney test was performed to compare average $\mathrm{ADC}$ values (ADCmin, $\mathrm{ADCmax}$, and ADCmean) between malignant and benign tumors in patients with head and neck cancer. And a value of $p<0.05$ was considered significant.

Three receiver operating characteristic (ROC) were 
curved to evaluate diagnostic capability of ADC values (ADCmin, ADCmax, and ADCmean) and to determine the cut-off value for differentiating malignant from benign nodes.

\section{Results}

Twenty-five patients (14 (56.0\%) males, 11 (44.0\%) females; mean age, 49.6 years) with 80 malignant and benign lymph nodes were included to the study referring to medical imaging center, located in Shohadaye Tajrish Hospital, Tehran, Iran between May 2017 and January 2018 (Figure 2, 3 and 4). The mean age of patients with benign and malignant tumors was equal to 51.70 and 46.97 , respectively. $41.7 \%$ of patients with benign tumors and $69.2 \%$ of patients with malignant tumors were male. The groups were similar in terms of age and gender (Table 1).

The mean short-axis diameter of the lymph nodes with metastasis was equal to $7.36 \pm 1.55 \mathrm{~mm}$ and for the non-metastasis was equal to $7.02 \pm 1.23 \mathrm{~mm}$. This difference was not statistically significant considering $\mathrm{p}$-value $=0.346$.

Upon pairwise comparison, the mean ADC values (ADCmin, ADCmax, and ADCmean) of benign tumors were significantly higher than the mean ADC values (ADCmin, ADCmax, and ADCmean) of malignant tumors (Table 2).

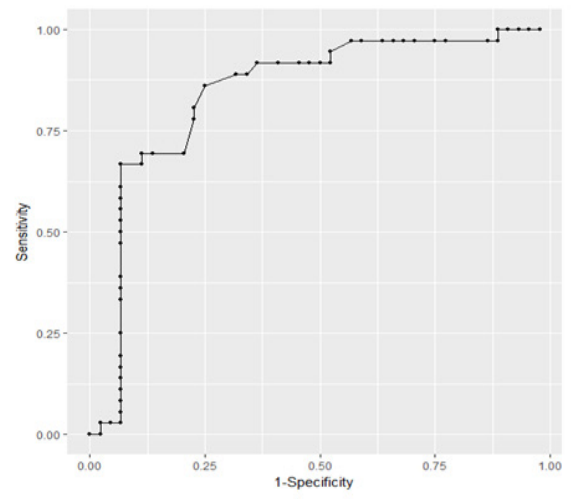

A

Table 1. Descriptive Statistics of Age and Gender

\begin{tabular}{lccc}
\hline Variable & Benign & Malignant & P-value \\
\hline Gender & & & \\
Male (\%) & $5(41.7 \%)$ & $9(69.2 \%)$ & $0.165^{*}$ \\
Female (\%) & $7(58.3 \%)$ & $4(30.8 \%)$ & \\
Mean Age (years) & $12(51.70 \pm 1.73)$ & $13(46.97 \pm 2.31)$ & $0.099^{* *}$ \\
\hline * Chi-Square Independent Test; ** Independent T-Test &
\end{tabular}

Table 2. Pairwise Comparison of Average ADC $\left(\times 10^{-3} \mathrm{~mm}^{2} / \mathrm{s}\right)$ Values between Groups

\begin{tabular}{lccc}
\hline Group & mean ADC a & $\min$ ADC a & $\max$ ADC a \\
\hline Benign & $1.06 \pm 0.12^{*}$ & $0.92 \pm 0.20$ & $1.24 \pm 0.15$ \\
Malignant & $0.90 \pm 0.10$ & $0.78 \pm 0.08$ & $1.02 \pm 0.12$ \\
P-value & $<0.001$ & 0.001 & $<0.001$ \\
\hline * mean \pm Standard Deviation,$\times 10^{-3} \mathrm{~mm}^{2} / \mathrm{s}$ &
\end{tabular}

Receiver operating characteristics (ROC) analysis indicated that ADC mean threshold value was lower than any values in the interval $\left[0.9936-0.9999 \times 10^{-3} \mathrm{~mm}^{2} / \mathrm{s}\right.$ ] that may differentiate malignant lesion from benign lesion (sensitivity by $80.0 \%$, specificity by $77 \%$, area under the curve (AUC) by0.79). Also, ADCmin threshold was any values in the interval $\left[0.8890-0.9099 \times 10^{-3} \mathrm{~mm}^{2} / \mathrm{s}\right]$ (sensitivityby $100.0 \%$, specificity by $64.0 \%$, AUC by 0.82 ) and ADCmax threshold was any values in the interval $\left[1.1199-1.1100 \times 10^{-3} \mathrm{~mm}^{2} / \mathrm{s}\right]$ (sensitivity by $80.0 \%$, specificity by $77.0 \%$, AUCby0.79) (Figure 1).

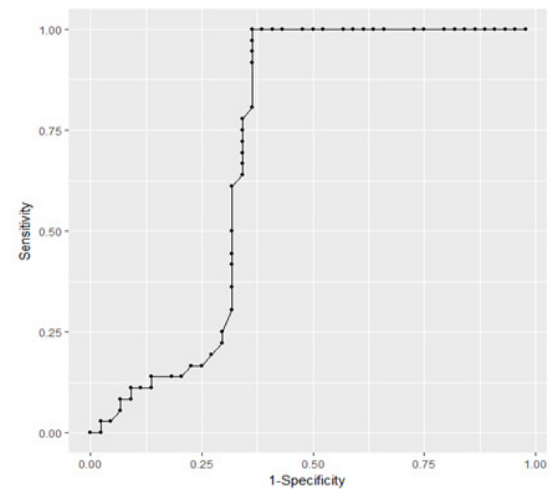

B

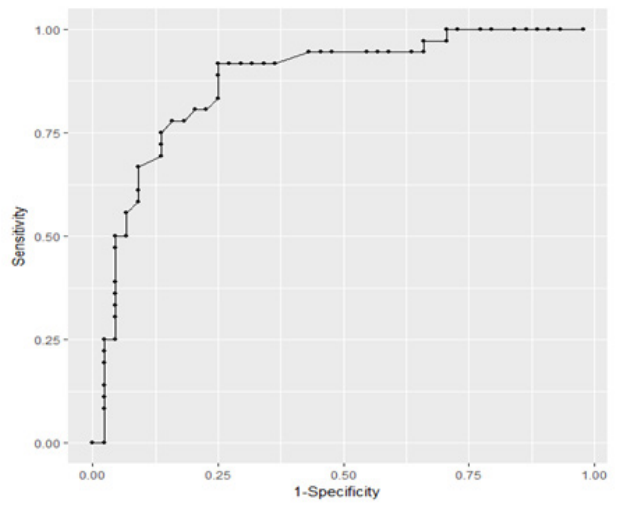

$\mathrm{C}$

Figure 1. ROC Curve for the A, ADCmean values; B, ADCmin; C, ADCmax for discrimination of malignant nodes from benign nodes. 

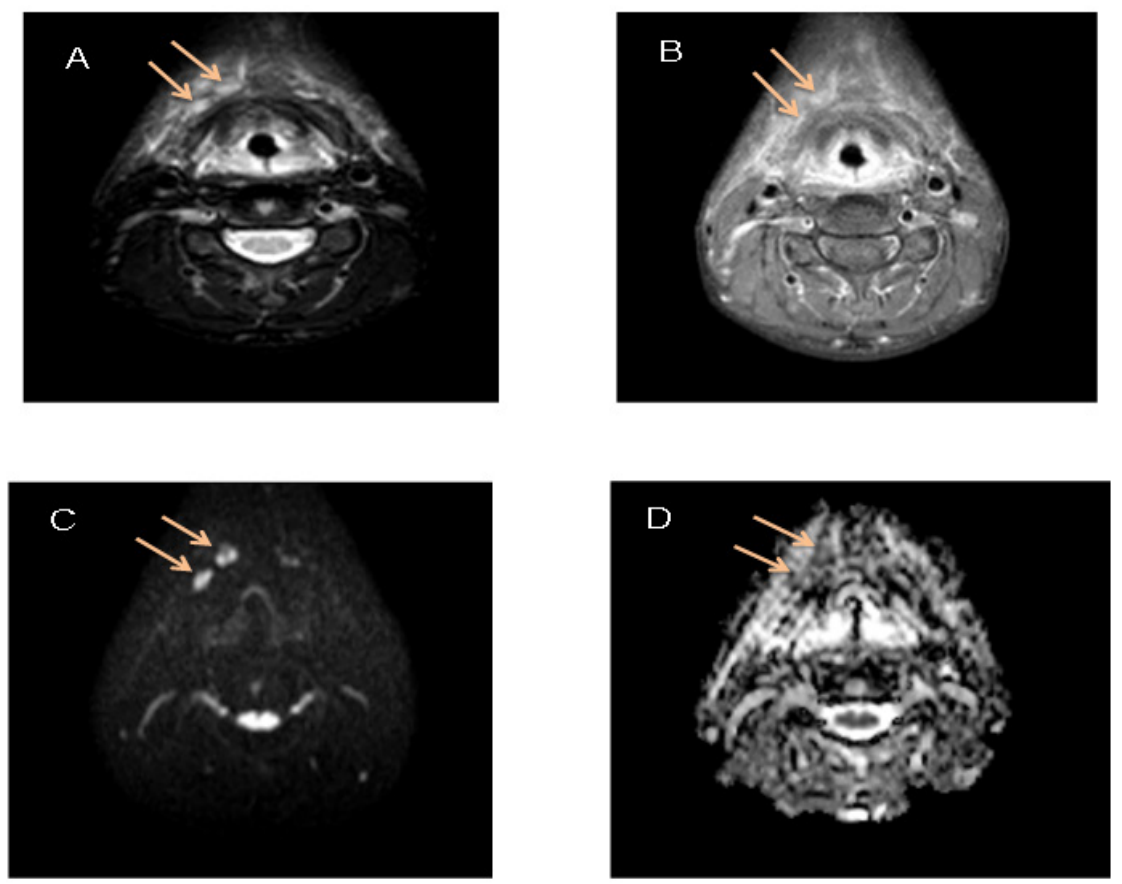

Figure 2. A 17 Year-Old Male with Thyroid Cancer. A, STIR shows two enlarged right neck LNs; B, T1W post contrast shows enhanced LNs; C, DWI shows high signal intensity; D, ADC displays restricted diffusion of LNs on $\operatorname{ADC}\left(0.865 \times 10^{-3} \mathrm{~mm}^{2} / \mathrm{s}\right)$.

\section{Discussion}

Predicting the status of cervical lymph nodes is crucial for diagnosis of malignancy, staging, therapy planning and follow-up in patients with Head and Neck cancer. While conventional imaging can detect enlarged neck lymph nodes, the differentiation between benign and malignant lymph nodes remains challenging as none of the morphological criteria, including size, shape or presence of necrosis is absolutely reliable( Ali, 2012; Ali and El
Hariri, 2017; Perrone et al., 2011).

In our study, the difference in the short axis diameter between metastatic $(7.36 \pm 1.55 \mathrm{~mm})$ and benign nodes $(7.02 \pm 1.23 \mathrm{~mm})$ is not statistically significant and there is an overlap in the diameter of the short axis between both groups ( $p$-value $=0.346$ ) that was similar to results of other studies (de Bondt et al., 2009; ElSaid et al., 2014). De Bondt (2009) and ElSaid (2014) stated, there is no significant relationship between the size of the lymph nodes and the presence of metastasis. Hence, the
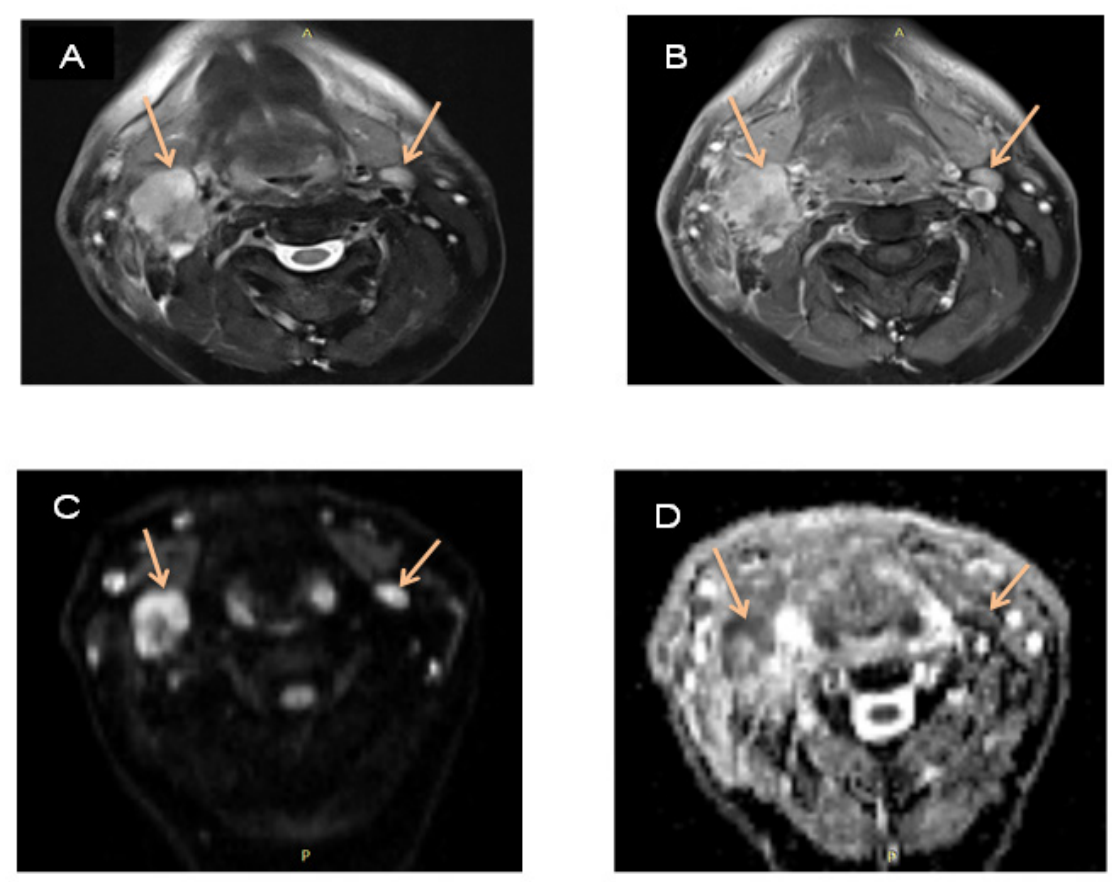

Figure 3. A 45 Year-Old Male with Thyroid and Larynx Cancer. A, T2-W fat sat shows two enlarged right and left neck LNs; B, T1W post contrast shows enhanced LNs; C, DWI shows high signal intensity; D, ADC displays restricted diffusion of LNs on ADC $\left(0.913 \times 10^{-3} \mathrm{~mm}^{2} / \mathrm{s}\right)$. 

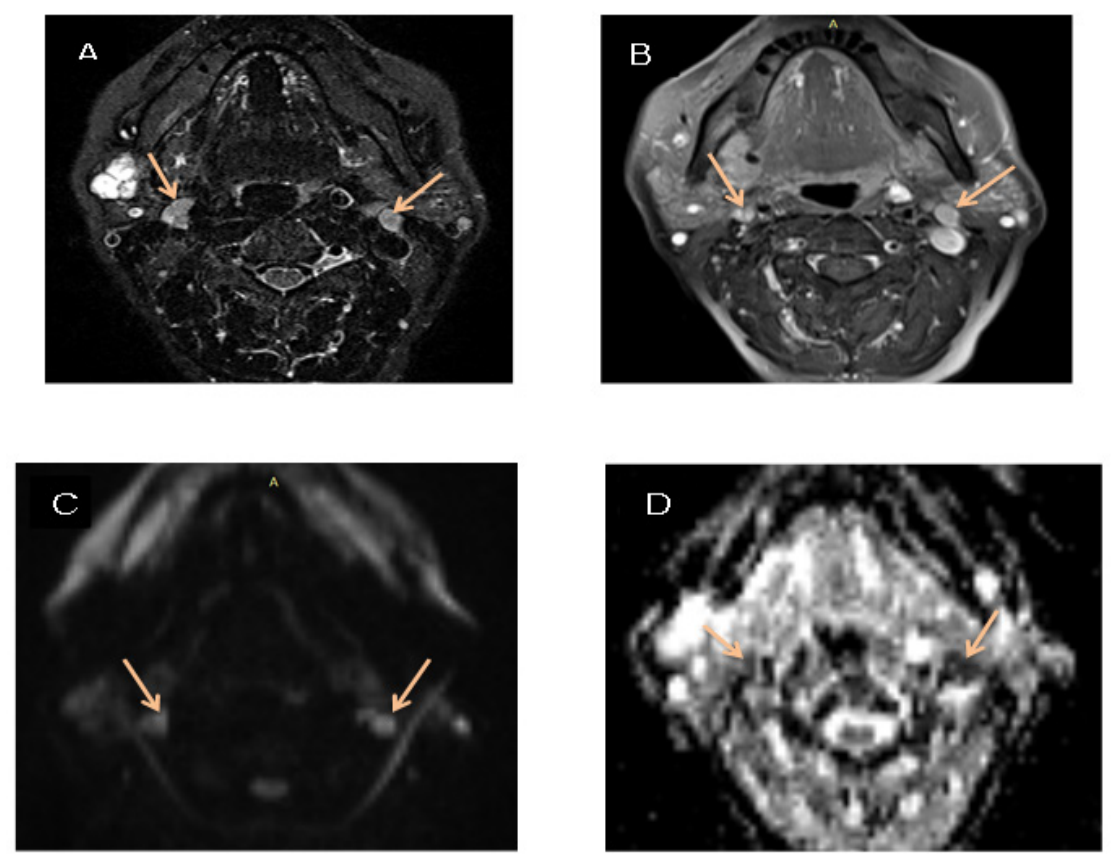

Figure 4. A 63 Year-Old Female with Parotid Mass. A, STIR shows two enlarged right and left neck LNs; B, T1W post contrast shows enhanced LNs; C, DWI shows high signal intensity; D, ADC displays restricted diffusion of LNs on $\operatorname{ADC}\left(0.883 \times 10^{-3} \mathrm{~mm}^{2} / \mathrm{s}\right)$.

differentiation between benign and malignant neck lymph nodes is not reliable through investigating morphological criteria such as the short axis diameter of lymph nodes (ElSaid et al., 2014).

In general, invasive methods such as: biopsy and dissection, and their side effects, as well as the low accuracy of other diagnostic imaging methods, such as ultrasound, CT scan, conventional MRI, and limitations of SPECT and PET imaging all have led researchers to make more effort in order to find a method that reduces the time, cost, and complicated diagnostic risks (Razek et al., 2006; Holzapfel et al., 2009; Salem et al., 2014; Liang et al., 2017).

Diffusion-weighted imaging probes local tissue microstructure reflected by the freedom of microscopic motion of water molecules. This motion depends on the size, the temperature, and in particular on the microscopic environment of the examined molecules. So, DWI is an unenhanced, simple, fast and non-invasive MRI technique that can provide information about characterization of LNs and differentiate benign LNs from malignant ones (Perrone et al., 2011; Zhang et al., 2013; Ragheb et al., 2014).

ADC is a quantitative parameter that is acquired through DWI images and can exclude the T2 shine-effect, also provide quantitative assessment of water diffusivity in the target tissues which makes it easier to differentiate between lesions (ElSaid et al., 2014; Salem et al., 2014; Hasanzadeh et al., 2017).

Similar to studies of (ElSaid et al., 2014; Ali and El Hariri, 2017) we also used high b-value of $1000 \mathrm{~s} / \mathrm{mm}^{2}$ to evaluate water diffusion more accurately and decrease the effects of capillary perfusion.

Our study included 25 patients with 80 enlarged neck LNs. They were 44 patients with benign lymphadenopathy,
36 patients with metastasis from head and neck cancer. The mean ADC values of the benign and metastasis groups were equal to $1.06 \pm 0.12 \times 10^{-3} \mathrm{~mm}^{2} / \mathrm{s}$ and 0.90 $\pm 0.10 \times 10^{-3} \mathrm{~mm}^{2} / \mathrm{s}$, respectively. The mean ADC values of the benign cervical lymph nodes were significantly higher than metastatic type that was similar to results of other studies ( Ali, 2012; Dawood et al., 2014; Razek et al., 2006; Salem et al., 2014; Liang et al., 2017; Perrone et al., 2011).

Ali et al., (2012) reported that the mean ADC value of metastatic lymph nodes $\left(0.92 \pm 0.13 \times 10^{-3} \mathrm{~mm}^{2} / \mathrm{s}\right)$ was significantly lower than mean ADC of benign cervical lymph nodes $\left(1.51 \pm 0.36 \times 10^{-3} \mathrm{~mm}^{2} / \mathrm{s}\right)(\mathrm{p}<0.0001)$.

Dawood et al., (2014) reported that ADC values of benign lymph nodes were significantly higher than those of malignant LNs, with a $p$ value $<0.0001$ and the mean $\mathrm{ADC}$ value for malignant lesions, that was equal to 0.90 $\pm 0.15 \times 10^{-3} \mathrm{~mm}^{2} / \mathrm{s}$, which is lower than that of benign LNs, that was equal to $1.52 \pm 0.37 \times 10^{-3} \mathrm{~mm}^{2} / \mathrm{s}$.

Razek et al., (2006) reported also a significantly higher mean ADC value in benign nodes (1.64 \pm 0.16 $\times 10^{-3} \mathrm{~mm}^{2} / \mathrm{s}$ ) compared to those of malignant nodes $\left(1.09 \pm 0.11 \times 10^{-3} \mathrm{~mm}^{2} / \mathrm{s}\right)$. Similar results was achieved by Perrone (2011) and they reported mean ADC values malignant lymph nodes by $0.85 \times 10^{-3} \mathrm{~mm}^{2} / \mathrm{s}$ versus $1.448 \times$ $10^{-3} \mathrm{~mm}^{2} / \mathrm{s}$ for benign nodes.

Few studies, such as Sumi et al., (2003) reported opposite results; in their studies, the ADC value of metastatic neck LNs was higher than that of non-metastatic one. These results may be explained by the difference in the histological types, the variation in lymph nodes at the cellular level, varying b-values and also heterogeneous ROI. An ROI may also include the necrotic and metastatic cells such that the central necrosis in examined metastatic lymph nodes (48\%) altered the ADC values of these nodes; 
and although low b-value, increases SNR, but reduces the diffusion sensitivity( Ali and El Hariri, 2017; Razek et al., 2006; Holzapfel et al., 2009; Perrone et al., 2011; Hasanzadeh et al., 2017).

In this study, we also measured the Min and max ADC values. Similarly to the results for the ADC mean, Min and max ADC values of metastatic neck LNs $(0.78 \pm 0.08 \times$ $10^{-3} \mathrm{~mm}^{2} / \mathrm{s}$ and $1.02 \pm 0.12 \times 10^{-3} \mathrm{~mm}^{2} / \mathrm{s}$ respectively) were lower than those of non-metastatic neck LNs $(0.92 \pm 0.20 \times$ $10^{-3} \mathrm{~mm}^{2} / \mathrm{s}$ and $1.24 \pm 0.15 \times 10^{-3} \mathrm{~mm}^{2} / \mathrm{s}$ respectively) .

In our study, the best cutoff value of ADC for distinguishing benign from malignant neck lymph nodes was equal to $0.996 \times 10^{-3} \mathrm{~mm}^{2} / \mathrm{s}$ with sensitivity, specificity and accuracy by $80.56 \%, 77.27 \%$ and 71.59 $\%$ respectively, which is approximately similar to the results of the studies which were conducted by de Bondt et al., (2009), Perrone et al., (2011), Taha Ali et al.,(2017), Elsaid et al.,(2014).

The corresponding value in the report of the study which was conducted by de Bondt et al., (2009) was equal to $1.0 \times 10^{-3} \mathrm{~mm}^{2} / \mathrm{s}$ with sensitivity and specificity by 92.3 and 83.9 respectively. Perrone et al., (2011) reported that the best cutoff value for discriminating malignant from benign nodes was equal to $1.03 \times 10^{-3} \mathrm{~mm}^{2} / \mathrm{s}$, obtaining a sensitivity by $100 \%$ and a specificity by $92.9 \%$. The best ADC threshold value in the study by Elsaid (2014) was equal to $1.005 \times 10^{-3} \mathrm{~mm}^{2} / \mathrm{s}$ with sensitivity by $100 \%$ and specificity by $62.5 \%$ for distinguishing benign from malignant neck lymph nodes. Taha Ali et al., (2017) reported that the optimal ADC threshold value was equal to $1.15 \times 10^{-3} \mathrm{~mm}^{2} / \mathrm{s}$ with sensitivity and specificity by $91.6 \%$ and $77.7 \%$, respectively for discriminating malignant from benign nodes.

In general, various studies obtained different values of mean and cutoff ADC for differentiating malignant from benign neck lymph nodes. The difference in these values can be explained by the fact that the ADC is influenced by various factors such as: different MR machines, MRI acquisition parameters, location, size and area of ROI, size of the lymph nodes as well as the body temperature (de Bondt et al., 2009; Perrone et al., 2011; Hasanzadeh et al., 2017).

Our study had some limitations. First: The DW-MRI method, due to spatial resolution limitations, has a low sensitivity in detecting lymph nodes which are less than $5 \mathrm{~mm}$. Therefore, in this study, neck lymph nodes which were less than $5 \mathrm{~mm}$ were not evaluated. In order to improve the resolution of diffusion images and achieve more anatomical details of the lesion and to examine lymph nodes which are smaller than $5 \mathrm{~mm}$, the study was suggested to use 3.0 T MRI. Second: trying to improve the diffusion sensitivity by increasing the $b$ value leads to the decline of the signal-to-noise ratio that limits the ADC measurement on the smaller lymph nodes. Third: DWI is sensitive to artifacts such as: motion, susceptibility and chemical shift which makes it difficult to discover lesions on DWI. A further limitation was the low number of patients included in the sample. Further studies can report better and more accurate results regarding DW-MRI, by choosing more samples.
In conclusion, the results of this study showed that MR diffusion imaging and ADC values as a non-invasive technique can assess metastatic neck LNs in head and neck cancer with higher sensitivity, specificity and accuracy.

\section{Conflict of Interest}

The authors do not have any possible conflicts of interest.

\section{Acknowledgments}

This study was approved by the local ethical committee of the university, and informed consent was obtained from the patients. Authors would like to thank Patients who participated in this study.

\section{References}

Dawood HA, Hassan TA, Mohey N (2014). Value of combined real time sonoelastography and apparent diffusion coefficient value measurement in differentiation of enlarged neck lymph nodes. Egypt J Radiol Nucl Med, 45, 387-94.

de Bondt RBJ, Hoeberigs MC, Nelemans PJ, et al (2009). Diagnostic accuracy and additional value of diffusion-weighted imaging for discrimination of malignant cervical lymph nodes in head and neck squamous cell carcinoma. Neuroradiology, 51, 183-92.

Dudau C, Hameed S, Gibson D, et al (2014). Can contrast-enhanced ultrasound distinguish malignant from reactive lymph nodes in patients with head and neck cancers?. Ultrasound Med Biol, 40, 747-54.

ElSaid NAE, Nada OMM, Habib YS, Semeisem AR, Khalifa NM (2014). Diagnostic accuracy of diffusion weighted MRI in cervical lymphadenopathy cases correlated with pathology results. Egypt J Radiol Nucl Med, 45, 1115-25.

Hasanzadeh F, Faeghi F, Valizadeh A, Bayani L (2017). Diagnostic value of diffusion weighted magnetic resonance imaging in evaluation of metastatic axillary lymph nodes in a sample of iranian women with breast cancer. Asian Pac J Cancer Prev, 18, 1265.

Holzapfel K, Duetsch S, Fauser C, et al (2009). Value of diffusion-weighted MR imaging in the differentiation between benign and malignant cervical lymph nodes. Eur J Radiol, 72, 381-7.

Kim S, Loevner L, Quon H, et al (2009). Diffusion weighted MRI for predicting and detecting early response to chemoradiation therapy of squamous cell carcinomas of the head and neck. Clin Cancer Res, 15, 986-94.

Liang L, Luo X, Lian Z, et al (2017). Lymph node metastasis in head and neck squamous carcinoma: Efficacy of intravoxel incoherent motion magnetic resonance imaging for the differential diagnosis. Eur J Radiol, 90, 159-65.

Mack MG, Rieger J, Baghi M, Bisdas S, Vogl TJ (2008). Cervical lymph nodes. Eur J Radiol, 66, 493-500.

Noij DP, Pouwels PJW, Ljumanovic R, et al (2015). Predictive value of diffusion-weighted imaging without and with including contrast-enhanced magnetic resonance imaging in image analysis of head and neck squamous cell carcinoma. Eur J Radiol, 84, 108-16.

Perrone A, Guerrisi P, Izzo L, et al (2011). Diffusion-weighted MRI in cervical lymph nodes: Differentiation between benign and malignant lesions. Eur J Radiol, 77, 281-6.

Ragheb AS, Abdel Rahman HM, Azeem Ismail AA (2014). Can diffusion weighted image and apparent diffusion coefficient (ADC) differentiate benign from malignant cervical 
adenopathy?. Egypt J Radiol Nucl Med, 45, 377-86.

Razek AAKA, Soliman NY, Elkhamary S, Alsharaway MK, Tawfik A (2006). Role of diffusion-weighted MR imaging in cervical lymphadenopathy. Eur Radiol, 16, 1468-77.

Salem F, Elshafey R, Elmahalawy M, Elshaeny S (2014). Apparent diffusion coefficient measurements in the differentiation between benign and malignant neck masses. Egypt J Radiol Nucl Med, 45, 367-75.

Sumi M, Sakihama N, Sumi T, et al (2003). Discrimination of metastatic cervical lymph nodes with diffusion-weighted MR imaging in patients with head and neck cancer. AJNR Am J Neuroradiol, 24, 1627.

Ali TF (2012). Neck lymph nodes: Characterization with diffusion-weighted MRI. Egypt J Radiol Nucl Med, 43, 173-81.

Ali TF, El Hariri MA (2017). Combined diffusion-weighted MRI and MR spectroscopy: Feasibility to improve the MRI capability in differentiation between benign and malignant neck lymphadenopathy. Egypt J Radiol Nucl Med, 48, 97-106.

Vandecaveye V, De Keyzer F, Vander Poorten V, et al (2009). Head and neck squamous cell carcinoma: value of diffusion-weighted MR imaging for nodal staging. Radiology, 251, 134-46.

Vandecaveye V, Dirix P, De Keyzer F, et al (2012). Diffusion-weighted magnetic resonance imaging early after chemoradiotherapy to monitor treatment response in head-and-neck squamous cell carcinoma. Int J Radiat Oncol Biol Phys, 82, 1098-107.

Zhang Y, Chen J, Shen J, et al (2013). Apparent diffusion coefficient values of necrotic and solid portion of lymph nodes: Differential diagnostic value in cervical lymphadenopathy. Clin Radiol, 68, 224-31.

\section{(c) (i) (8)}

This work is licensed under a Creative Commons AttributionNon Commercial 4.0 International License. 